\title{
Existence of Square-Mean Almost Automorphic Solutions to Stochastic Functional Integrodifferential Equations in Hilbert Spaces
}

\author{
Lijie Li \\ School of Mathematics and Information Sciences, Yulin Normal University, Yulin 537000, China \\ Correspondence should be addressed to Lijie Li; lilijie1219@126.com
}

Received 20 December 2013; Accepted 13 February 2014; Published 27 March 2014

Academic Editor: Yonghuia Xia

Copyright (C) 2014 Lijie Li. This is an open access article distributed under the Creative Commons Attribution License, which permits unrestricted use, distribution, and reproduction in any medium, provided the original work is properly cited.

\begin{abstract}
The existence and uniqueness of square-mean almost automorphic mild solution to a stochastic functional integrodifferential equation is studied. Under some appropriate assumptions, the existence and uniqueness of square-mean almost automorphic mild solution is obtained by Banach's fixed point theorem. Particularly, based on Schauder's fixed point theorem, the existence of squaremean almost automorphic mild solution is obtained by using the condition which is weaker than Lipschitz conditions. Finally, an example illustrating our main result is given.
\end{abstract}

\section{Introduction}

The almost periodic type solutions to stochastic differential equations are among the most attractive topics in mathematical analysis due to their extensive applications in areas such as physics, economics, mathematical biology, and engineering. And the concept of almost automorphic functions, which was initially introduced in the literature by Bochner [1], is an important generalization of the almost periodic functions. From then on, the almost automorphic functions and the almost automorphic solutions for differential systems have been investigated by many mathematicians [2-13].

Integrodifferential equations arose naturally in mechanics, electromagnetic theory, heat flow, nuclear reactor dynamics, and population dynamics [14]. The papers $[5,14-20]$ are concerned with the existence of almost periodic type solutions to stochastic functional integrodifferential equations. Ding et al. [21] investigated the existence of pseudo almost periodic solutions for an equation arising in the study of heat conduction in materials with memory, which could be transformed into the following abstract integrodifferential equation:

$$
u^{\prime}(t)=A u(t)+\int_{0}^{t} B(t-s) u(s) d s+f(t, u(t)), \quad t \geq 0 .
$$

Diagana et al. [5] established the existence and uniqueness of asymptotically almost automorphic mild solution to an abstract partial neutral integrodifferential equation with unbounded delay

$$
\begin{gathered}
\frac{d}{d t} D\left(t, u_{t}\right)=A D\left(t, u_{t}\right)+\int_{0}^{t} B(t-s) D\left(s, u_{s}\right) d s \\
+g\left(t, u_{t}\right), \quad t \in[\sigma, \sigma+a), \\
u_{\sigma}=\varphi,
\end{gathered}
$$

where $D(t, \varphi)=\varphi(0)+f(t, \varphi)$.

Furthermore, noise or stochastic perturbation is unavoidable and omnipresent in nature as well as in man-made systems. This paper is mainly focused on the existence and uniqueness of square-mean almost automorphic mild solutions to the following stochastic functional integrodifferential equations in the abstract form:

$$
\begin{aligned}
& d N(t, x(t))= A N(t, x(t)) d t+\int_{0}^{t} B(t-s) N(s, x(s)) d s d t \\
&+h\left(t, x\left(\gamma_{2}(t)\right)\right) d t+f\left(t, x\left(\gamma_{3}(t)\right)\right) d W(t), \\
& t \geq 0
\end{aligned}
$$


where $A: D(A) \subseteq L^{2}(P, H) \rightarrow L^{2}(P, H), B(t) \quad:$ $D(B(t)) \subseteq L^{2}(P, H) \rightarrow L^{2}(P, H)$, and $t \geq 0$ are linear, closed, and densely defined operators on $L^{2}(P, H)$ and $W(t)$ is a two-sided standard one-dimensional Brownian motion defined on the filtered probability space $\left(\Omega, \mathscr{F}, P, \mathscr{F}_{t}\right)$, where $\mathscr{F}_{t}=\sigma\{W(u)-W(v) ; u, v \leq t\}$. Here $N(t, x(t))=x(t)-$ $a\left(t, x\left(\gamma_{1}(t)\right)\right), a, h, f$, and $\gamma_{i}(i=1,2,3)$ are appropriate functions to be specified later.

The Lipschitz condition is a very important condition in the field of the existence and uniqueness of solutions for differential equations. In [15], Chang et al. established a new composition theorem for square-mean almost automorphic functions under conditions which were different from Lipschitz conditions in the literature. And they apply this new composition theorem to investigate the existence of square-mean almost automorphic mild solutions for a stochastic differential equation. It can be proved that the conditions in [15] (see assumption (H3)(ii)) are weaker than Lipschitz condition. In other words, a function with Lipschitz condition is satisfied (H3)(ii). However, there exists such a function which satisfies (H3)(ii) but does not satisfy Lipschitz condition (see Remark 11).

In this paper, firstly, upon using Lipschitz condition and other some appropriate assumptions, some sufficient conditions for the existence and uniqueness of square-mean almost automorphic mild solution to (3) are given. Secondly, by virtue of new composition theorem in [15] together with Schauder's fixed point theorem, we investigate the existence of square-mean almost automorphic mild solutions for a stochastic differential equation in a real separable Hilbert space, which is different from Lipschitz condition in the literature. Finally, we discuss the existence and uniqueness of an almost automorphic mild solution to a concrete integrodifferential equation, which is an illustration to demonstrate our main analyses.

\section{Preliminaries}

Throughout this paper, we assume that $(H,\|\cdot\|,\langle\cdot, \cdot\rangle)$ and $\left(K,\|\cdot\|_{K},\langle\cdot, \cdot\rangle_{K}\right)$ are two real separable Hilbert spaces. Let $(\Omega, \mathscr{F}, P)$ be a complete probability space. The notation $L^{2}(P, H)$ stands for the space of all $H$-valued random variables $x$ such that

$$
E\|x\|^{2}=\int_{\Omega}\|x\|^{2} d P<\infty .
$$

For $x \in L^{2}(P, H)$, let

$$
\|x\|_{2}=\left(\int_{\Omega}\|x\|^{2} d P\right)^{1 / 2} .
$$

Then it is routine to check that $L^{2}(P, H)$ is a Hilbert space equipped with the norm $\|\cdot\|_{2}$. The notations $C\left(R ; L^{2}(P, H)\right)$ and $B C\left(R ; L^{2}(P, H)\right)$ stand for the collection of all continuous stochastic processes from $R$ into $L^{2}(P, H)$ and the space of all bounded continuous stochastic processes $x: R \rightarrow L^{2}(P, H)$, respectively. It is then easy to check that $B C\left(R ; L^{2}(P, H)\right)$ is a Banach space when it is endowed with the norm
$\|x\|_{B C\left(R ; L^{2}(P, H)\right)}:=\sup _{t \in R}\|x(t)\|_{2}$. Let $L(K, H)$ denote the space of all linear bounded operators from $K$ into $H$, which are equipped with the usual operator norm $\|\cdot\|_{L(K, H)}$; in particular, it is simply denoted by $\mathscr{L}(H)$ when $K=H$. In addition, $W(t)$ is a two-sided standard one-dimensional Brownian motion defined on the filtered probability space $\left(\Omega, \mathscr{F}, P, \mathscr{F}_{t}\right)$, where $\mathscr{F}_{t}=\sigma\{W(u)-W(v) ; u, v \leq t\}$.

Definition 1 (see [18]). A family of bounded linear operators $T(t): t \geq 0$ from $L^{2}(P, H)$ into $L^{2}(P, H)$ is a resolvent operator family for the problem

$$
\begin{aligned}
& d x(t)=A x(t) d t+\int_{0}^{t} B(t-s) x(s) d s d t, \quad t \geq 0, \\
& x(0)=x_{0} \in L^{2}(P, H), \quad t \geq 0,
\end{aligned}
$$

if the following conditions are satisfied:

(a) $T(0)=I$ (the identity operator on $L^{2}(P, H)$ ) and the function $T(t) x$ is continuous on $[0,+\infty)$ for every $x \in$ $L^{2}(P, H)$;

(b) $T(t) D(A) \subseteq D(A)$ for all $t \geq 0$ and for $x \in$ $D(A), A T(t) x$ is continuous on $[0,+\infty)$, and $T(t) x$ is continuously differentiable on $[0,+\infty)$;

(c) for $x \in D(A)$, the following resolvent equations hold:

$$
\begin{aligned}
& \frac{d}{d t} T(t) x=A T(t) x+\int_{0}^{t} B(t-s) T(s) x d s, \quad t \geq 0 \\
& \frac{d}{d t} T(t) x=T(t) A x+\int_{0}^{t} T(t-s) A B(s) x d s, \quad t \geq 0 .
\end{aligned}
$$

For more details on semigroup theory and resolvent operators, we refer the reader to $[16,18,22]$.

Definition 2 (see [17]). A stochastic process $x: R \rightarrow L^{2}(P$, $H$ ) is said to be stochastically continuous if

$$
\lim _{t \rightarrow s} E\|x(t)-x(s)\|^{2}=0 .
$$

Definition 3 (see [17]). A stochastically continuous stochastic process $x: R \rightarrow L^{2}(P, H)$ is said to be square-mean almost automorphic if, for every sequence of real numbers, there exists a subsequence $\left\{s_{n}\right\}_{n \in N}$ and a stochastic process $y: R \rightarrow$ $L^{2}(P, H)$ such that

$$
\begin{aligned}
& \lim _{n \rightarrow \infty} E\left\|x\left(t+s_{n}\right)-y(t)\right\|^{2}=0, \\
& \lim _{n \rightarrow \infty} E\left\|y\left(t-s_{n}\right)-x(t)\right\|^{2}=0
\end{aligned}
$$

for each $t \in R$. The collection of all square-mean almost automorphic stochastic processes $x: R \rightarrow L^{2}(P, H)$ is denoted by $A A\left(R ; L^{2}(P, H)\right)$.

Definition 4. A function $f: R \times L^{2}(P, H) \rightarrow L^{2}(P, H)$, $(t, x) \rightarrow f(t, x)$, which is jointly continuous, is said to be square-mean almost automorphic if $f(t, x)$ is square-mean almost automorphic in $t \in R$ uniformly for all $x \in K$, where 
$K$ is any bounded subset of $L^{2}(P, H)$. That is to say, for every sequence of real numbers $\left\{s_{n}^{\prime}\right\}_{n \in N}$, there exist a subsequence $\left\{s_{n}\right\}_{n \in N}$ and a function $\tilde{f}: R \times L^{2}(P, H) \rightarrow L^{2}(P, H)$ such that

$$
\begin{aligned}
& \lim _{n \rightarrow \infty} E\left\|f\left(t+s_{n}, x\right)-\tilde{f}(t, x)\right\|^{2}=0, \\
& \lim _{n \rightarrow \infty} E\left\|\tilde{f}\left(t-s_{n}, x\right)-f(t, x)\right\|^{2}=0
\end{aligned}
$$

for each $t \in R$ and each $x \in K$.

Lemma 5 (see [17]). $\left(A A\left(R ; L^{2}(P, H)\right),\|\cdot\|_{\infty}\right)$ is a Banach space when it is equipped with the norm

$$
\|\cdot\|_{\infty}:=\sup _{t \in R}\|x(t)\|^{2}=\sup _{t \in R}\left(E\|x(t)\|^{2}\right)^{1 / 2}
$$

for $x \in A A\left(R ; L^{2}(P, H)\right)$.

Lemma 6 (see [17]). If $x, x_{1}$, and $x_{2}$ are all square-mean almost automorphic stochastic processes, then the following hold:

(i) $x_{1}+x_{2}$ is square-mean almost automorphic;

(ii) $\lambda x$ is square-mean almost automorphic for every scalar $\lambda$;

(iii) there exists a constant $M>0$ such that $\sup _{t \in R}\|x(t)\|_{2} \leq$ $M$. That is, $x$ is bounded in $L^{2}(P, H)$.

Theorem 7 (see [17]). Let $f: R \times L^{2}(P, H) \rightarrow L^{2}(P, H)$, $(t, x) \rightarrow f(t, x)$ be square-mean almost automorphic in $t \in R$ for each $x \in L^{2}(P, H)$, and assume that $f$ satisfies a Lipschitz condition in the following sense:

$$
E\|f(t, x)-f(t, y)\|^{2} \leq M E\|x-y\|^{2}
$$

for all $x, y \in L^{2}(P, H)$ and for each $t \in R$, where $M>$ 0 is independent of $t$. Then for any square-mean almost automorphic process $x: R \rightarrow L^{2}(P, H)$, the stochastic process $F: R \rightarrow L^{2}(P, H)$ given by $F(\cdot)=f(\cdot, x(\cdot))$ is square-mean almost automorphic.

Theorem 8 (see [15]). Let $f: R \times L^{2}(P, H) \rightarrow L^{2}(P, H)$, $(t, x) \rightarrow f(t, x)$ be square-mean almost automorphic, and assume that $f(t, \cdot)$ is uniformly continuous on each bounded subset $K \subset L^{2}(P, H)$ uniformly for $t \in R$; that is, for all $\varepsilon>0$, there exists $\delta>0$ such that $x, y \in K$ and $E\|x-y\|^{2}<\delta$ imply that $E\|f(t, x)-f(t, y)\|^{2}<\varepsilon$ for all $t \in R$. Then for any square-mean almost automorphic process $x: R \rightarrow L^{2}(P, H)$, the stochastic process $F: R \rightarrow L^{2}(P, H)$ given by $F(\cdot):=$ $f(\cdot, x(\cdot))$ is square-mean almost automorphic.

\section{Some Lemmas}

In this section, we apply Theorems 7 and 8 to investigate the existence of square-mean almost automorphic mild solutions to problem (3).
Definition 9. A stochastically continuous stochastic process $x:[0, \infty) \rightarrow L^{2}(P, H)$ is called a mild solution of the system (3) if $x(0)=x_{0}$ and $x(t)$ satisfies

$$
\begin{aligned}
x(t)= & T(t)\left[x_{0}-a\left(0, x\left(\gamma_{1}(0)\right)\right)\right]+a\left(t, x\left(\gamma_{1}(t)\right)\right) \\
& +\int_{0}^{t} T(t-s) h\left(s, x\left(\gamma_{2}(s)\right)\right) d s \\
& +\int_{0}^{t} T(t-s) f\left(s, x\left(\gamma_{3}(s)\right)\right) d W(s)
\end{aligned}
$$

for all $t \geq 0$.

Remark 10. It is easy to see that if $T(t)$ is exponentially stable, then we obtain that the stochastic process $x$ is a mild solution to problem (3) if and only if $x$ satisfies the stochastic integral equation

$$
\begin{aligned}
x(t)= & a\left(t, x\left(\gamma_{1}(t)\right)\right)+\int_{-\infty}^{t} T(t-s) h\left(s, x\left(\gamma_{2}(s)\right)\right) d s \\
& +\int_{-\infty}^{t} T(t-s) f\left(s, x\left(\gamma_{3}(s)\right)\right) d W(s)
\end{aligned}
$$

for all $t \geq 0$.

Let us list the following assumptions.

(H1) There exists a resolvent operator $T(\cdot)$ of $(3)$, and $T(\cdot)$ is exponentially stable; that is,

$$
\|T(t)\| \leq M e^{-\delta t}
$$

for all $t \geq 0$ and some constants $M, \delta>0$. Moreover, $T(t)$ is compact for $t \geq 0$.

(H2) The functions $a, h, f \in A A\left(R^{+} \times L^{2}(P, H), L^{2}(P, H)\right)$ and there exist positive numbers $l_{a}, l_{h}, l_{f}$ such that

$$
\begin{aligned}
& E\|a(t, x)-a(t, y)\|^{2} \leq l_{a} E\|x-y\|^{2}, \\
& E\|h(t, x)-h(t, y)\|^{2} \leq l_{h} E\|x-y\|^{2}, \\
& E\|f(t, x)-f(t, y)\|^{2} \leq l_{f} E\|x-y\|^{2}
\end{aligned}
$$

for all $t \geq 0$ and each $x, y \in L^{2}(P, H)$.

(H3) $\gamma_{i}(\cdot) \in C\left(R^{+}, R^{+}\right), i=1,2,3$.

(H4) The functions $a, h, f: R^{+} \times L^{2}(P, H) \rightarrow L^{2}(P, H)$ satisfy the following conditions.

(i) $a, h, f$ are square-mean almost automorphic and $a(t, \cdot), f(t, \cdot), g(t, \cdot)$ are uniformly continuous in every uniformly bounded subset $K \subset$ $L^{2}(P, H)$ for $t \geq 0$.

(ii) There exist integrable functions $m_{a}, m_{h}, m_{f}$ : $R \rightarrow[0, \infty)$ and continuous nondecreasing 
functions $W_{a}, W_{h}, W_{f}:[0, \infty) \rightarrow(0, \infty)$ such that

$$
\begin{aligned}
& E\|a(t, \varphi)\|^{2} \leq m_{a}(t) W_{a}\left(E\|\varphi\|^{2}\right), \\
& E\|f(t, \varphi)\|^{2} \leq m_{h}(t) W_{h}\left(E\|\varphi\|^{2}\right), \\
& E\|g(t, \varphi)\|^{2} \leq m_{f}(t) W_{f}\left(E\|\varphi\|^{2}\right)
\end{aligned}
$$

for all $(t, \varphi) \in R^{+} \times K$.

(iii) Let $x_{n} \quad \subset \quad A A\left(R^{+} ; L^{2}(P, H)\right)$ be uniformly bounded in $R^{+}$and uniformly convergent in each compact subset of $R^{+}$. Then $a\left(\cdot, x_{n}(\cdot)\right)$, $h\left(\cdot, x_{n}(\cdot)\right)$, and $f\left(\cdot, x_{n}(\cdot)\right)$ are relatively compact in $B C\left(R^{+} ; L^{2}(P, H)\right)$.

Remark 11. Obviously, when the continuous function $f$ satisfies Lipschitz condition like (H2) and $f(t, 0)=0$, then function $f$ satisfies (H4)(ii). However, the function $f(t, x)=$ $\sqrt{x}[\sin t+\sin \sqrt{2} t]$ satisfies (H4)(ii) but does not satisfy (H2).

In the proof of the existence theorem, we need the following technical lemmas.

Lemma 12. Assume that conditions (H1)-(H3) hold. Let $L$ be the operator defined by, for each $x \in A A\left(R^{+} ; L^{2}(P, H)\right)$,

$$
\begin{aligned}
(L x)(t):= & a\left(t, x\left(\gamma_{1}(t)\right)\right)+\int_{-\infty}^{t} T(t-s) h\left(s, x\left(\gamma_{2}(s)\right)\right) d s \\
& +\int_{-\infty}^{t} T(t-s) f\left(s, x\left(\gamma_{3}(s)\right)\right) d W(s) \\
:= & a\left(t, x\left(\gamma_{1}(t)\right)\right)+\Phi x(t)+\Psi x(t) .
\end{aligned}
$$

\section{Then $L$ maps $A A\left(R^{+} ; L^{2}(P, H)\right)$ into itself.}

Proof. Firstly, let $x \in A A\left(R^{+} ; L^{2}(P, H)\right)$; then $s \rightarrow x\left(\gamma_{1}(s)\right)$ is in $A A\left(R^{+} ; L^{2}(P, H)\right)$ as $\gamma_{1}(\cdot) \in C\left(R^{+}, R^{+}\right)$. Hence, by $(\mathrm{H} 2)$ and Theorem 7 , one can easily see that $s \rightarrow a\left(s, x\left(\gamma_{1}(s)\right)\right)$ belongs to $A A\left(R^{+} ; L^{2}(P, H)\right)$.

Similarly, $s \rightarrow h\left(s, x\left(\gamma_{2}(s)\right)\right)$ and $s \rightarrow f\left(s, x\left(\gamma_{3}(s)\right)\right)$ belong to $A A\left(R^{+} ; L^{2}(P, H)\right)$ whenever $x \in A A\left(R^{+} ; L^{2}(P, H)\right)$.

Secondly, we show that $\Phi$ is square-mean almost automorphic.

Let $\left\{s_{n}^{\prime}\right\}_{n \in N}$ be an arbitrary sequence of real numbers. Since $H(\cdot)=h\left(\cdot, x\left(\gamma_{2}(\cdot)\right)\right) \in A A\left(R^{+} ; L^{2}(P, H)\right)$, there exists a subsequence $\left\{s_{n}\right\}_{n \in N}$ of $\left\{s_{n}^{\prime}\right\}_{n \in N}$ such that, for a certain stochastic process $\widetilde{H}$,

$$
\begin{aligned}
& \lim _{n \rightarrow \infty} E\left\|H\left(t+s_{n}\right)-\widetilde{H}(t)\right\|^{2}=0, \\
& \lim _{n \rightarrow \infty} E\left\|\widetilde{H}\left(t-s_{n}\right)-H(t)\right\|^{2}=0
\end{aligned}
$$

hold for each $t \geq 0$. Moreover, if we let $\widetilde{\Phi} x(t)=\int_{-\infty}^{t} T(t-$ $s) \widetilde{H}(s) d s$, by using Cauchy-Schwarz inequality, we have

$$
\begin{aligned}
E \| & \Phi\left(t+s_{n}\right)-\widetilde{\Phi} x(t) \|^{2} \\
= & E\left\|\int_{-\infty}^{t+s_{n}} T\left(t+s_{n}-s\right) H(s) d s-\int_{-\infty}^{t} T(t-s) \widetilde{H}(s) d s\right\|^{2} \\
\leq & E\left(\int_{-\infty}^{t}\left\|T(t-s)\left[H\left(s+s_{n}\right)-\widetilde{H}(s)\right]\right\| d s\right)^{2} \\
\leq & M^{2} E\left(\int_{-\infty}^{t} e^{-\delta(t-s)}\left\|H\left(s+s_{n}\right)-\widetilde{H}(s)\right\| d s\right)^{2} \\
\leq & M^{2}\left(\int_{-\infty}^{t} e^{-\delta(t-s)} d s\right) \\
& \times\left(\int_{-\infty}^{t} e^{-\delta(t-s)} E\left\|H\left(s+s_{n}\right)-\widetilde{H}(s)\right\|^{2} d s\right)^{2} \\
\leq & M^{2}\left(\int_{-\infty}^{t} e^{-\delta(t-s)} d s\right)^{2} \sup _{t \geq 0} E\left\|H\left(t+s_{n}\right)-\widetilde{H}(t)\right\|^{2} \\
\leq & \frac{M^{2}}{\delta^{2}} \sup _{t \geq 0} E\left\|H\left(t+s_{n}\right)-\widetilde{H}(t)\right\|^{2} .
\end{aligned}
$$

Thus, by (19), we immediately obtain that

$$
\lim _{n \rightarrow \infty} E\left\|\Phi x\left(t+s_{n}\right)-\widetilde{\Phi} x(t)\right\|^{2}=0
$$

for each $t \geq 0$. And we can show in a similar way that

$$
\lim _{n \rightarrow \infty} E\left\|\widetilde{\Phi} x\left(t-s_{n}\right)-\Phi x(t)\right\|^{2}=0
$$

for each $t \geq 0$. Thus we conclude that $\Phi x(t) \in A A\left(R^{+} ; L^{2}(P\right.$, $H)$ ). phic.

Thirdly, we show that $\Psi$ is square-mean almost automor-

Since $F(\cdot)=f\left(\cdot, x\left(\gamma_{3}(\cdot)\right)\right) \in A A\left(R^{+} ; L^{2}(P, H)\right)$, there exists a subsequence $\left\{s_{n}\right\}_{n \in N}$ of $\left\{s_{n}^{\prime}\right\}_{n \in N}$ such that, for a certain stochastic process $\widetilde{F}$,

$$
\begin{aligned}
& \lim _{n \rightarrow \infty} E\left\|F\left(t+s_{n}\right)-\widetilde{F}(t)\right\|^{2}=0, \\
& \lim _{n \rightarrow \infty} E\|\| \widetilde{F}\left(t-s_{n}\right)-F(t) \|^{2}=0
\end{aligned}
$$

hold for each $t \geq 0$.

This is more complicated than the previous case because of the involvement of the Brownian motion $W$. To overcome such a difficulty, we make extensive use of the properties of $\widetilde{W}$ defined by

$$
\widetilde{W}(\sigma):=W\left(\sigma+s_{n}\right)-W\left(s_{n}\right)
$$


for each $\sigma$. Note that $\widetilde{W}$ is a Brownian motion and has the same distribution as $W$. Moreover, if we let $\widetilde{\Psi} x(t)=\int_{-\infty}^{t} T(t-$ s) $\widetilde{F}(s) d s$, then, by making a change of variable $\sigma=s-s_{n}$, we get

$$
\begin{aligned}
& E \| \Psi x\left(t+s_{n}\right)-\widetilde{\Psi} x(t) \|^{2} \\
&=E \| \int_{-\infty}^{t+s_{n}} T\left(t+s_{n}-s\right) F(s) d W(s) \quad-\int_{-\infty}^{t} T(t-s) \widetilde{F}(s) d W(s) \|^{2} \\
&=E\left\|\int_{-\infty}^{t} T(t-\sigma)\left[F\left(\sigma+s_{n}\right)-\widetilde{F}(\sigma)\right] d \widetilde{W}(\sigma)\right\|^{2} .
\end{aligned}
$$

Then, using an estimate on the Ito integral established, we obtain

$$
\begin{aligned}
& E\left\|\Psi x\left(t+s_{n}\right)-\widetilde{\Psi} x(t)\right\|^{2} \\
& \quad \leq E\left(\int_{-\infty}^{t}\left\|T(t-\sigma)\left[F\left(\sigma+s_{n}\right)-\widetilde{F}(\sigma)\right]\right\|^{2} d \sigma\right) \\
& \quad \leq M^{2} \int_{-\infty}^{t} e^{-2 \delta(t-\sigma)} E\left\|F\left(\sigma+s_{n}\right)-\widetilde{F}(\sigma)\right\|^{2} d \sigma \\
& \quad \leq \frac{M^{2}}{2 \delta} \sup _{t \geq 0} E\left\|F\left(t+s_{n}\right)-\widetilde{F}(t)\right\|^{2} .
\end{aligned}
$$

Thus, by (23), we immediately obtain that

$$
\lim _{n \rightarrow \infty} E\left\|\Psi x\left(t+s_{n}\right)-\widetilde{\Psi} x(t)\right\|^{2}=0
$$

for each $t \geq 0$. And we can show in a similar way that

$$
\lim _{n \rightarrow \infty} E\left\|\widetilde{\Psi} x\left(t-s_{n}\right)-\Psi x(t)\right\|^{2}=0
$$

for each $t \geq 0$. Thus we conclude that $\Psi x(t) \in A A\left(R^{+} ; L^{2}(P\right.$, $H)$ ).

According to Lemma 6, we can easily obtain that $L$ maps $A A\left(R^{+} ; L^{2}(P, H)\right)$ into itself.

Lemma 13. Assume that conditions (H1), (H3), and (H4)(i) are satisfied. Then $L$ is continuous and maps $A A\left(R^{+} ; L^{2}(P, H)\right)$ into itself, where L is defined by Lemma 12.

\section{Proof.}

Step 1. We prove that $L$ maps $A A\left(R^{+} ; L^{2}(P, H)\right)$ into itself.

Firstly, let $x \in A A\left(R^{+} ; L^{2}(P, H)\right)$; then $s \rightarrow x\left(\gamma_{1}(s)\right)$ is in $A A\left(R^{+} ; L^{2}(P, H)\right)$ as $\gamma_{1}(\cdot) \in C\left(R^{+}, R^{+}\right)$. Hence, by (H4)(i) and Theorem 8 , one can easily see that $s \rightarrow a\left(s, x\left(\gamma_{1}(s)\right)\right)$ belongs to $A A\left(R^{+} ; L^{2}(P, H)\right)$.

Similarly, $s \rightarrow h\left(s, x\left(\gamma_{2}(s)\right)\right)$ and $s \rightarrow f\left(s, x\left(\gamma_{3}(s)\right)\right)$ belong to $A A\left(R^{+} ; L^{2}(P, H)\right)$ whenever $x \in A A\left(R^{+} ; L^{2}(P, H)\right)$.

Secondly, from Lemma 12 , we can prove that $\Phi$ and $\Psi$ are square-mean almost automorphic.

According to Lemma 6, we can easily obtain that $L$ is continuous and maps $A A\left(R^{+} ; L^{2}(P, H)\right)$ into itself.

Step 2. We prove that $L$ is continuous on $A A\left(R^{+} ; L^{2}(P, H)\right)$.
Let $\left\{x_{n}\right\} \subset\left(R^{+} ; L^{2}(P, H)\right)$ be a sequence which converges to some $x \in\left(R^{+} ; L^{2}(P, H)\right)$ with respect to $\|\cdot\|_{\infty}$; that is, $\left\|x_{n}-x\right\|_{\infty} \rightarrow 0$ as $n \rightarrow \infty$. There exists a bounded subset $K \subset L^{2}(P, H)$ such that $x_{n}(t), x(t) \subset K$ for $t \geq 0, n=1,2, \ldots$. By (H3), (H4)(i), and Theorem 8 , for any $\varepsilon>0$, there exist $\delta>0$ and $N>0$ such that $E\left\|x_{n}\left(\gamma_{i}(t)\right)-x\left(\gamma_{i}(t)\right)\right\|^{2}<\delta(i=$ $1,2,3)$, which imply that

$$
\begin{gathered}
E\left\|a\left(t, x_{n}\left(\gamma_{1}(t)\right)\right)-a\left(t, x\left(\gamma_{1}(t)\right)\right)\right\|^{2}<\frac{\varepsilon}{9}, \\
E\left\|h\left(t, x_{n}\left(\gamma_{2}(t)\right)\right)-h\left(t, x\left(\gamma_{2}(t)\right)\right)\right\|^{2}<\frac{\delta^{2} \varepsilon}{9 M^{2}}, \\
E\left\|f\left(t, x_{n}\left(\gamma_{3}(t)\right)\right)-f\left(t, x\left(\gamma_{3}(t)\right)\right)\right\|^{2}<\frac{2 \delta \varepsilon}{9 M^{2}}
\end{gathered}
$$

for all $n>N$ and $t \geq 0$, where $\delta, M$ are given in (H1). Then

$$
\begin{aligned}
& E\left\|\left(L x_{n}\right)(t)-(L x)(t)\right\|^{2} \\
& \leq 3 E\left\|a\left(t, x_{n}\left(\gamma_{1}(t)\right)\right)-a\left(t, x\left(\gamma_{1}(t)\right)\right)\right\|^{2} \\
& +3 E\left(\int_{-\infty}^{t} \| T(t-s)\left[h\left(t, x_{n}\left(\gamma_{2}(t)\right)\right)\right.\right. \\
& \left.\left.-h\left(t, x\left(\gamma_{2}(t)\right)\right)\right] \| d s\right)^{2} \\
& +3 E \| \int_{-\infty}^{t} T(t-s) \\
& \times\left[f\left(t, x_{n}\left(\gamma_{3}(t)\right)\right)-f\left(t, x\left(\gamma_{3}(t)\right)\right)\right] d W(s) \|^{2} \\
& \leq \frac{\varepsilon}{3}+3 M^{2}\left(\int_{-\infty}^{t} e^{-\delta(t-s)} d s\right) \\
& \times\left(\int_{-\infty}^{t} e^{-\delta(t-s)} E\left\|h\left(t, x_{n}\left(\gamma_{2}(t)\right)\right)-h\left(t, x\left(\gamma_{2}(t)\right)\right)\right\|^{2} d s\right) \\
& +3 M^{2} \int_{-\infty}^{t} e^{-2 \delta(t-s)} E\left\|f\left(t, x_{n}\left(\gamma_{3}(t)\right)\right)-f\left(t, x\left(\gamma_{3}(t)\right)\right)\right\|^{2} d s \\
& \leq \frac{\varepsilon}{3}+3 M^{2}\left(\int_{-\infty}^{t} e^{-\delta(t-s)} d s\right)\left(\int_{-\infty}^{t} e^{-\delta(t-s)} \frac{\delta^{2} \varepsilon}{9 M^{2}} d s\right) \\
& +3 M^{2}\left(\int_{-\infty}^{t} e^{-2 \delta(t-s)} \frac{2 \delta \varepsilon}{9 M^{2}} d s\right) \\
& \leq \frac{\varepsilon}{3}+\frac{\delta^{2} \varepsilon}{3}\left(\int_{-\infty}^{t} e^{-\delta(t-s)} d s\right)^{2} \\
& +\frac{2 \delta \varepsilon}{3} \int_{-\infty}^{t} e^{-2 \delta(t-s)} d s=\varepsilon
\end{aligned}
$$

for all $n>N$ and all $t \geq 0$. This implies that $L$ is continuous. The proof is completed. 


\section{Existence of Square-Mean Almost Automorphic Solutions}

Theorem 14. If the assumptions (H1)-(H3) are satisfied, then the system (3) has a unique square-mean almost periodic automorphic mild solution, whenever $\Theta<1 / 3$, where

$$
\Theta=l_{a}+\frac{M^{2}}{\delta^{2}} l_{h}+\frac{M^{2}}{2 \delta} l_{f} .
$$

Proof. Let $L: A A\left(R^{+}, L^{2}(P, H)\right) \rightarrow C\left(R^{+}, L^{2}(P, H)\right)$ be the operator defined by

$$
\begin{aligned}
L x(t):= & a\left(t, x\left(\gamma_{1}(t)\right)\right)+\int_{-\infty}^{t} T(t-s) h\left(s, x\left(\gamma_{2}(s)\right)\right) d s \\
& +\int_{-\infty}^{t} T(t-s) f\left(s, x\left(\gamma_{3}(s)\right)\right) d W(s) .
\end{aligned}
$$

From the proof of Lemma 12, we see that $H(\cdot), F(\cdot) \in$ $A A\left(R^{+}, L^{2}(P, H)\right)$. Therefore, $L x(t) \in A A\left(R^{+}, L^{2}(P, H)\right)$; thus $L x(\cdot)$ maps $A A\left(R^{+}, L^{2}(P, H)\right)$ into itself.

To complete the proof, it suffices to prove that $L x(\cdot)$ is a contraction. Since $(a+b+c)^{2} \leq 3 a^{2}+3 b^{2}+3 c^{2}$, for $x(t), y(t) \in$ $A A\left(R^{+}, L^{2}(P, H)\right)$, we obtain

$$
\begin{aligned}
& E\|L x(t)-L y(t)\|^{2} \\
& \leq 3 E\left\|a\left(t, x\left(\gamma_{1}(t)\right)\right)-a\left(t, y\left(\gamma_{1}(t)\right)\right)\right\|^{2} \\
& +3 E\left\|\int_{-\infty}^{t} T(t-s)\left[h\left(s, x\left(\gamma_{2}(s)\right)\right)-h\left(s, y\left(\gamma_{2}(s)\right)\right)\right] d s\right\|^{2} \\
& +3 E \| \int_{-\infty}^{t} T(t-s)\left[f\left(s, x\left(\gamma_{3}(s)\right)\right)\right. \\
& \left.\quad-f\left(s, y\left(\gamma_{3}(s)\right)\right)\right] d W(s) \|^{2}
\end{aligned}
$$

Evaluating the three terms of the right-hand side, we have

$$
\begin{aligned}
& 3 E\left\|a\left(t, x\left(\gamma_{1}(t)\right)\right)-a\left(t, y\left(\gamma_{1}(t)\right)\right)\right\|^{2} \\
& \leq 3 l_{a} \sup _{s \geq 0} E\left\|x\left(\gamma_{2}(s)\right)-y\left(\gamma_{2}(s)\right)\right\|^{2} \leq 3 l_{a}\|x-y\|_{\infty}^{2}, \\
& 3 E\left\|\int_{-\infty}^{t} T(t-s)\left[h\left(s, x\left(\gamma_{2}(s)\right)\right)-h\left(s, y\left(\gamma_{2}(s)\right)\right)\right] d s\right\|^{2} \\
& \leq 3 M^{2}\left(\int_{-\infty}^{t} e^{-\delta(t-s)} d s\right) \\
& \quad \times\left(\int_{-\infty}^{t} e^{-\delta(t-s)} E\left\|h\left(s, x\left(\gamma_{2}(s)\right)\right)-h\left(s, y\left(\gamma_{2}(s)\right)\right)\right\|^{2} d s\right)
\end{aligned}
$$

$$
\begin{aligned}
\leq & 3 M^{2} l_{h}\left(\int_{-\infty}^{t} e^{-\delta(t-s)} d s\right) \\
& \times\left(\int_{-\infty}^{t} e^{-\delta(t-s)} E\left\|x\left(\gamma_{2}(s)\right)-y\left(\gamma_{2}(s)\right)\right\|^{2} d s\right) \\
& \leq 3 M^{2} l_{h}\left(\int_{-\infty}^{t} e^{-\delta(t-s)} d s\right)^{2} \sup _{s \geq 0} E\left\|x\left(\gamma_{2}(s)\right)-y\left(\gamma_{2}(s)\right)\right\|^{2} \\
& \leq \frac{3 M^{2}}{\delta^{2}} l_{h}\|x-y\|_{\infty}^{2}, \\
3 & \left\|\int_{-\infty}^{t} T(t-s)\left[f\left(s, x\left(\gamma_{3}(s)\right)\right)-f\left(s, y\left(\gamma_{3}(s)\right)\right)\right] d W(s)\right\|^{2} \\
& \leq 3 M^{2} \int_{-\infty}^{t} e^{-2 \delta(t-s)} E\left\|f\left(s, x\left(\gamma_{3}(s)\right)\right)-f\left(s, y\left(\gamma_{3}(s)\right)\right)\right\|^{2} d s \\
& \leq 3 M^{2} l_{f}\left(\int_{-\infty}^{t} e^{-2 \delta(t-s)} d s\right) \sup _{s \geq 0} E\left\|x\left(\gamma_{3}(s)\right)-y\left(\gamma_{3}(s)\right)\right\|^{2} \\
\leq & \frac{3 M^{2}}{2 \delta} l_{f}\|x-y\|_{\infty}^{2} .
\end{aligned}
$$

Thus, we obtain that

$$
\begin{aligned}
& E\|L x(t)-L y(t)\|^{2} \\
& \quad \leq\left(3 l_{a}+\frac{3 M^{2}}{\delta^{2}} l_{h}+\frac{3 M^{2}}{2 \delta} l_{f}\right)\|x-y\|_{\infty}^{2}=3 \Theta\|x-y\|_{\infty}^{2}
\end{aligned}
$$

for each $t \geq 0$

Consequently, if $\Theta<1 / 3$, then (3) has a unique fixed point, which is the unique square-mean almost periodic automorphic mild solution to (3), such that $L x(t)=x(t)$.

Theorem 15. Assume that conditions (H1), (H3), and (H4) are satisfied; then problem (3) admits at least one square-mean almost automorphic mild solution on $R$ provided that

$$
\begin{gathered}
L_{h}=\sup _{t \geq 0} \int_{-\infty}^{t} e^{-\delta(t-s)} m_{h}(s) d s<\infty, \\
L_{f}=\sup _{t \geq 0} \int_{-\infty}^{t} e^{-2 \delta(t-s)} m_{f}(s) d s<\infty, \\
m_{a}(t) \liminf _{r \rightarrow \infty} \frac{W_{a}(r)}{r}+\frac{M^{2} L_{h}}{\delta} \liminf _{r \rightarrow \infty} \frac{W_{h}(r)}{r} \\
+M^{2} L_{f} \liminf _{r \rightarrow \infty} \frac{W_{f}(r)}{r}<\frac{1}{3} .
\end{gathered}
$$

Proof. For the sake of convenience, we break the proof into several steps.

Step 1. $L$ is continuous.

From the proof of the previous lemmas it is clear that the nonlinear operator $L$ is well defined and continuous. Moreover, by Lemma 13 , we infer that $L x \in A A\left(R^{+} ; L^{2}(P, H)\right)$ 
whenever $x \in A A\left(R^{+} ; L^{2}(P, H)\right)$; that is, $L$ maps $A A\left(R^{+}\right.$; $\left.L^{2}(P, H)\right)$ into itself.

Step 2. $L$ maps bounded sets into bounded sets.

Let $B_{r}=\left\{x \in A A\left(R^{+} ; L^{2}(P, H)\right):\|x\|_{\infty} \leq r\right\}$ for each $r>$ 0 . We prove that there exists a number $r$ such that $L\left(B_{r}\right) \subseteq B_{r}$.

Clearly, for each positive number $r, B_{r}$ is a bounded closed convex set in $A A\left(R^{+} ; L^{2}(P, H)\right)$. We claim that there exists a positive number $r$ such that $L\left(B_{r}\right) \subseteq B_{r}$. If it is not true, then for every $r>0$ there exist $x_{r}(\cdot) \in B_{r}$ and $t_{r} \geq 0$ such that $E\left\|\left(L x_{r}\right)\left(t_{r}\right)\right\|^{2}>r$. However, on the other hand, we have

$$
\begin{aligned}
r< & E\left\|\left(L x_{r}\right)\left(t_{r}\right)\right\|^{2} \\
\leq & 3 E\left\|a\left(t_{r}, x\left(\gamma_{1}\left(t_{r}\right)\right)\right)\right\|^{2} \\
& +3 E\left\|\int_{-\infty}^{t_{r}} T\left(t_{r}-s\right) h\left(s, x\left(\gamma_{2}(s)\right)\right) d s\right\|^{2} \\
& +3 E\left\|\int_{-\infty}^{t_{r}} T\left(t_{r}-s\right) f\left(s, x\left(\gamma_{3}(s)\right)\right) d W(s)\right\|^{2} \\
\leq & 3 m_{a}(t) W_{a}(r) \\
& +3 M^{2}\left[\left(\int_{-\infty}^{t_{r}} e^{-\delta\left(t_{r}-s\right)} d s\right)\right. \\
& \left.\times\left(\int_{-\infty}^{t_{r}} e^{-\delta\left(t_{r}-s\right)} E\left\|h\left(s, x\left(\gamma_{2}(s)\right)\right)\right\|^{2} d s\right)\right] \\
& +3 M^{2}\left(\int_{-\infty}^{t_{r}} e^{-2 \delta\left(t_{r}-s\right)} E\left\|f\left(s, x\left(\gamma_{3}(s)\right)\right)\right\|^{2} d s\right) \\
\leq & 3 m_{a}(t) W_{a}(r)+\frac{3 M^{2}}{\delta} \int_{-\infty}^{t_{r}} e^{-\delta\left(t_{r}-s\right)} m_{h}(s) W_{h}(r) d s \\
& +3 M^{2} \int_{-\infty}^{t_{r}} e^{-2 \delta\left(t_{r}-s\right)} m_{f}(s) W_{f}(r) d s \\
\leq & 3 m_{a}(t) W_{a}(r)+\frac{3 M^{2} L_{h}}{\delta} W_{h}(r)+3 M^{2} L_{f} W_{f}(r) .
\end{aligned}
$$

Dividing both sides by $r$ and taking the lower limit as $r \rightarrow$ $\infty$, we obtain

$$
\begin{aligned}
1 \leq & 3 m_{a}(t) \liminf _{r \rightarrow \infty} \frac{W_{a}(r)}{r}+\frac{3 M^{2} L_{h}}{\delta} \liminf _{r \rightarrow \infty} \frac{W_{h}(r)}{r} \\
& +3 M^{2} L_{f} \liminf _{r \rightarrow \infty} \frac{W_{f}(r)}{r},
\end{aligned}
$$

which contradicts condition (37). Thus, for some positive number $r, L\left(B_{r}\right) \subseteq B_{r}$.

Step 3. $L$ maps bounded sets into equicontinuous sets.

Next we prove that the operator $L$ is completely continuous on $B_{r}$. It suffices to prove that the following statements are true.

(i) $V(t)=\left\{(L x)(t): x \in B_{r}\right\}$ is relatively compact in $L^{2}(P, H)$ for each $t \geq 0$. (ii) $\left\{L x: x \in B_{r}\right\} \subset A A\left(R^{+} ; L^{2}(P, H)\right)$ is a family of equicontinuous functions.

Firstly, we show that (i) holds. Let $0<\varepsilon<1$ be given. For each $t \geq 0$ and $x \in B_{r}$, we define

$$
\begin{aligned}
\left(L_{\varepsilon} x\right)(t)= & a\left(t-\varepsilon, x\left(\gamma_{1}(t-\varepsilon)\right)\right)+T(\varepsilon) \\
& \times\left[\int_{-\infty}^{t-\varepsilon} T(t-\varepsilon-s) h\left(s, x\left(\gamma_{2}(s)\right)\right) d s\right. \\
& \left.\quad+\int_{-\infty}^{t-\varepsilon} T(t-\varepsilon-s) f\left(s, x\left(\gamma_{3}(s)\right)\right) d W(s)\right] \\
= & a\left(t-\varepsilon, x\left(\gamma_{1}(t-\varepsilon)\right)\right) \\
& +T(\varepsilon)[(\Phi x)(t-\varepsilon)+(\Psi x)(t-\varepsilon)] .
\end{aligned}
$$

Since $T(t)(t \geq 0)$ is compact and (H4)(iii), then the set $V_{\varepsilon}(t)=\left\{\left(L_{\varepsilon} x\right)(t): x \in B_{r}\right\}$ is relatively compact in $L^{2}(P, H)$ for each $t \geq 0$. Moreover, for every $x \in B_{r}$, we have

$$
\begin{aligned}
& E\left\|(L x)(t)-\left(L_{\varepsilon} x\right)(t)\right\|^{2} \\
& \leq 3 E\left\|a\left(t, x\left(\gamma_{1}(t)\right)\right)-a\left(t-\varepsilon, x\left(\gamma_{1}(t-\varepsilon)\right)\right)\right\|^{2} \\
& \quad+3 E\left\|\int_{t-\varepsilon}^{t} T(t-s) h\left(s, x\left(\gamma_{2}(s)\right)\right) d s\right\|^{2} \\
& \quad+3 E\left\|\int_{t-\varepsilon}^{t} T(t-s) f\left(s, x\left(\gamma_{3}(s)\right)\right) d W(s)\right\|^{2} \\
& \leq 3 E\left\|a\left(t, x\left(\gamma_{1}(t)\right)\right)-a\left(t-\varepsilon, x\left(\gamma_{1}(t-\varepsilon)\right)\right)\right\| \\
& \quad+3 M^{2}\left(\int_{t-\varepsilon}^{t} e^{-\delta(t-s)} d s\right)^{2} \sup _{t \geq 0} E\|h(t)\|^{2} \\
& \quad+3 M^{2}\left(\int_{t-\varepsilon}^{t} e^{-2 \delta(t-s)} d s\right) \sup _{t \geq 0} E\|f(t)\|^{2} .
\end{aligned}
$$

Observe that $a\left(\cdot, x\left(\gamma_{1}(\cdot)\right)\right)$ is continuous; therefore, letting $\varepsilon \rightarrow 0$, there are relatively compact sets $V_{\varepsilon}(t)$ arbitrarily close to $V(t)$ and hence $V(t)$ is also relatively compact in $L^{2}(P, H)$ for each $t \geq 0$ and $L$ is completely continuous on $B_{r}$. Then

We now show that (ii) holds. Let $t_{1}, t_{2} \geq 0, t_{1}<t_{2}, x \in B_{r}$.

$$
\begin{aligned}
& E\left\|(L x)\left(t_{2}\right)-(L x)\left(t_{1}\right)\right\|^{2} \\
& \leq E \| a\left(t_{2}, x\left(\gamma_{1}\left(t_{2}\right)\right)\right)-a\left(t_{1}, x\left(\gamma_{1}\left(t_{1}\right)\right)\right) \\
& \quad+\int_{-\infty}^{t_{2}} T\left(t_{2}-s\right) h\left(s, x\left(\gamma_{2}(s)\right)\right) d s \\
& \quad-\int_{-\infty}^{t_{1}} T\left(t_{1}-s\right) h\left(s, x\left(\gamma_{2}(s)\right)\right) d s \\
& \quad+\int_{-\infty}^{t_{2}} T\left(t_{2}-s\right) f\left(s, x\left(\gamma_{3}(s)\right)\right) d W(s) \\
& \quad-\int_{-\infty}^{t_{1}} T\left(t_{1}-s\right) f\left(s, x\left(\gamma_{3}(s)\right)\right) d W(s) \|^{2}
\end{aligned}
$$




$$
\begin{aligned}
& \leq 4 E\left\|a\left(t_{2}, x\left(\gamma_{1}\left(t_{2}\right)\right)\right)-a\left(t_{1}, x\left(\gamma_{1}\left(t_{1}\right)\right)\right)\right\|^{2} \\
& +4 E\left\|\int_{t_{1}}^{t_{2}} T\left(t_{2}-t_{1}\right) T\left(t_{1}-s\right) h\left(s, x\left(\gamma_{2}(s)\right)\right) d s\right\|^{2} \\
& +4 E\left\|\int_{t_{1}}^{t_{2}} T\left(t_{2}-t_{1}\right) T\left(t_{1}-s\right) f\left(s, x\left(\gamma_{3}(s)\right)\right) d W(s)\right\|^{2} \\
& +4 E \|\left(T\left(t_{2}-t_{1}\right)-I\right) \\
& \quad \times\left(\int_{-\infty}^{t_{1}} T\left(t_{1}-s\right) h\left(s, x\left(\gamma_{2}(s)\right)\right) d s\right. \\
& \left.\quad-\int_{-\infty}^{t_{1}} T\left(t_{1}-s\right) f\left(s, x\left(\gamma_{3}(s)\right)\right) d W(s)\right) \|^{2} \\
& \leq 4 E\left\|a\left(t_{2}, x\left(\gamma_{1}\left(t_{2}\right)\right)\right)-a\left(t_{1}, x\left(\gamma_{1}\left(t_{1}\right)\right)\right)\right\|^{2} \\
& +4 M^{2}\left(\int_{t_{1}}^{t_{2}} e^{-\delta\left(t_{2}-s\right) d s}\right)^{2} \sup _{t \geq 0}\|h(t)\|^{2} \\
& +4 M^{2}\left(\int_{t_{1}}^{t_{2}} e^{-2 \delta\left(t_{2}-s\right) d s}\right) \sup _{t \geq 0}\|f(t)\|^{2} \\
& +4 \sup _{y \in V(t)} E\left\|\left(T\left(t_{2}-t_{1}\right)-I\right) y\right\|^{2} .
\end{aligned}
$$

The right-hand side tends to 0 independently of $x \in B_{r}$ as $t_{1} \rightarrow t_{2}$, which implies that the set $\left\{L x: x \in B_{r}\right\}$ is right equicontinuous at $t$. By a similar procedure we can show that $\left\{L x: x \in B_{r}\right\}$ is left equicontinuous at $t$. Thus, the set $\{L x$ : $x \in B_{r}$ \} is equicontinuous; that is, $L$ maps $B_{r}$ into a family of equicontinuous functions.

As a consequence of Steps 1-3 together with Schauder's fixed point theorem, we deduce that $L$ has a fixed point in $B_{r}$ which is a square-mean almost periodic mild solution to (3). The proof is completed.

\section{Example}

Consider the neutral stochastic partial functional integrodifferential equations of the form

$$
\begin{aligned}
d N(t, x(t))= & A N(t, x(t)) d t+\int_{0}^{t} B(t-s) N(s, x(s)) d s d t \\
& +h\left(t, x\left(\gamma_{2}(t)\right)\right) d t+f\left(t, x\left(\gamma_{3}(t)\right)\right) d W(t),
\end{aligned}
$$

for $(t, \xi) \in[0, \infty) \times[0, \pi]$, where $W(t)$ is a two-sided standard one-dimensional Brownian motion defined on the filtered probability space $\left(\Omega, \mathscr{F}, P, \mathscr{F}_{t}\right), \mathscr{F}_{t}=\sigma\{W(u)-W(v) ; u, v \leq$ $t\}$. Here $N(t, x(t))=x(t)-a\left(t, x\left(\gamma_{1}(t)\right)\right)$ and $\gamma_{1}(t)=\gamma_{2}(t)=$ $\gamma_{3}(t)=\lambda t+\lambda_{0}$, the constants $\lambda>0, \lambda_{0}>0$.

For that, let $\Omega \subset R^{2}$ be an open subset whose boundary $\partial \Omega$ is sufficiently regular. Let $X=H_{0}^{1}(\Omega) \times L^{2}(\Omega)$ and consider the linear operator $A$ whose domain is given by $D(A)=\left(H^{2}(\Omega) \bigcap H_{0}^{1}(\Omega)\right) \times H_{0}^{1}(\Omega)$ and

$$
A\left(\begin{array}{l}
x \\
y
\end{array}\right)=\left(\begin{array}{c}
\alpha^{\prime \prime}(0) x^{\prime \prime}-\beta(0) y
\end{array}\right),
$$

where $\alpha(\cdot), \beta(\cdot)$ are real-valued functions of class $C^{2}$ on $[0, \infty)$ such that $\alpha(0)>0$, and $\beta(0)>0$.

From Chen [23], we see that $A$ is the infinitesimal generator of a uniformly exponentially stable $C_{0}$-semigroup $(\widetilde{T}(t))_{t \geq 0}$ on $H_{0}^{1}(\Omega) \times L^{2}(\Omega)$. In what follows, we will assume that $M, \delta>0$ are positive constants such that $\|\widetilde{T}(t)\| \leq M e^{-2 \delta t}$ for all $t>0$.

Let $B(t)=A F(t)$, where $F: H_{0}^{1}(\Omega) \times L^{2}(\Omega) \rightarrow H_{0}^{1}(\Omega) \times$ $L^{2}(\Omega)$ is the operator family defined by

$$
F=\left(F_{i j}\right)=\left(\begin{array}{cc}
0 & 0 \\
-\beta^{\prime}(t)+\beta(0) \frac{\alpha^{\prime}(t)}{\alpha(0)} & \frac{\alpha^{\prime}(t)}{\alpha(0)}
\end{array}\right),
$$

and assume that

$$
\begin{gathered}
\max \left\{\left\|F_{22}(t)\right\|,\left\|F_{21}(t)\right\|\right\} \leq \frac{\delta}{\widetilde{M}} e^{-2 \delta t}, \quad t \geq 0, \\
\max \left\{\left\|F_{22}^{\prime}(t)\right\|,\left\|F_{21}^{\prime}(t)\right\|\right\} \leq \frac{\delta^{2}}{\widetilde{M}^{2}} e^{-2 \delta t}, \quad t \geq 0 .
\end{gathered}
$$

From the results in Grimmer [22], we see that the abstract integrodifferential system,

$$
x^{\prime}(t)=A x(t)+\int_{0}^{t} A F(t-s) x(s) d s,
$$

has an associated uniformly exponentially stable resolvent of operators $\{T(t)\}_{t \geq 0}$ with $\|T(t)\| \leq M e^{-\delta t}$ for $t \geq 0$.

Let

$$
\begin{aligned}
& a(t, \varphi)(\xi)=\beta \varphi(\xi)[\sin t+\sin \sqrt{2} t], \\
& h(t, \varphi)(\xi)=\beta \varphi(\xi)[\sin t+\sin \sqrt{3} t], \\
& f(t, \varphi)(\xi)=\beta \varphi(\xi)[\sin t+\sin \sqrt{5} t] .
\end{aligned}
$$

Thus, one has

$$
\begin{aligned}
& E\|a(t, x)-a(t, y)\|^{2} \leq 4 \beta^{2}\|x-y\|^{2}, \\
& E\|h(t, x)-h(t, y)\|^{2} \leq 4 \beta^{2}\|x-y\|^{2}, \\
& E\|f(t, x)-f(t, y)\|^{2} \leq 4 \beta^{2}\|x-y\|^{2} .
\end{aligned}
$$

It follows that $l_{a}=l_{h}=l_{f}=4 \beta^{2}$. Therefore, according to Theorem 14, (43) has a unique square-mean almost periodic mild solution provided that $\beta<\delta / \sqrt{6\left(2 \delta^{2}+2 M^{2}+M^{2} \delta\right)}$. Let

$$
\begin{aligned}
& a(t, \varphi)(\xi)=\beta \sin (\varphi(\xi))[\sin t+\sin \sqrt{2} t], \\
& h(t, \varphi)(\xi)=\beta \sin (\varphi(\xi))[\sin t+\sin \sqrt{3} t], \\
& f(t, \varphi)(\xi)=\beta \sin (\varphi(\xi))[\sin t+\sin \sqrt{5} t] .
\end{aligned}
$$


Thus, one has

$$
\begin{aligned}
& E\|a(t, \varphi)\|^{2} \leq \beta^{2}[\sin t+\sin \sqrt{2} t]^{2} E\|\varphi(\xi)\|^{2}, \\
& E\|h(t, \varphi)\|^{2} \leq \beta^{2}[\sin t+\sin \sqrt{3} t]^{2} E\|\varphi(\xi)\|^{2}, \\
& E\|f(t, \varphi)\|^{2} \leq \beta^{2}[\sin t+\sin \sqrt{5} t]^{2} E\|\varphi(\xi)\|^{2} .
\end{aligned}
$$

It follows that

$$
\begin{aligned}
& m_{a}(t)=\beta^{2}[\sin t+\sin \sqrt{2} t]^{2}, \\
& m_{h}(t)=\beta^{2}[\sin t+\sin \sqrt{3} t]^{2}, \\
& m_{f}(t)=\beta^{2}[\sin t+\sin \sqrt{5} t]^{2}
\end{aligned}
$$

and $W_{a}(r)=W_{h}(r)=W_{f}(r)=r$.

Obviously,

$$
\begin{aligned}
L_{h} & =\sup _{t \geq 0} \int_{-\infty}^{t} e^{-\delta(t-s)} m_{h}(s) d s \\
& =\beta^{2} \sup _{t \geq 0} \int_{-\infty}^{t} e^{-\delta(t-s)}(\sin t+\sin \sqrt{3} t)^{2} d s<\infty, \\
L_{f} & =\sup _{t \geq 0} \int_{-\infty}^{t} e^{-2 \delta(t-s)} m_{f}(s) d s \\
& =\beta^{2} \sup _{t \geq 0} \int_{-\infty}^{t} e^{-2 \delta(t-s)}(\sin t+\sin \sqrt{5} t)^{2} d s<\infty .
\end{aligned}
$$

Let

$$
\begin{aligned}
& \widetilde{L}_{a}=\sup _{t \geq 0}(\sin t+\sin \sqrt{2} t)^{2}, \\
& \widetilde{L}_{h}=\sup _{t \geq 0} \int_{-\infty}^{t} e^{-\delta(t-s)}(\sin t+\sin \sqrt{3} t)^{2} d s, \\
& \widetilde{L}_{f}=\sup _{t \geq 0} \int_{-\infty}^{t} e^{-2 \delta(t-s)}(\sin t+\sin \sqrt{5} t)^{2} d s .
\end{aligned}
$$

Therefore, according to Theorem 15, (43) has a square-mean almost periodic mild solution provided that

$$
\beta<\sqrt{\frac{\delta}{3\left(\delta \widetilde{L}_{a}+M^{2} \widetilde{L}_{h}+\delta M^{2} \widetilde{L}_{f}\right)}} .
$$

\section{Conflict of Interests}

The author declares that there is no conflict of interests regarding the publication of this paper.

\section{Acknowledgments}

This work was supported by the National Natural Science Foundation of China (no. 11161051), the Scientific Research Foundation of the Higher Education Institutions of Guangxi Province of China (no. 201202ZD080) and the Scientific Research Foundation of Guangxi Education Office (no. 201012MS183).

\section{References}

[1] S. Bochner, "A new approach to almost periodicity," Proceedings of the National Academy of Sciences of the United States of America, vol. 48, pp. 2039-2043, 1962.

[2] P. H. Bezandry and T. Diagana, "Existence of quadratic-mean almost periodic solutions to some stochastic hyperbolic differential equations," Electronic Journal of Differential Equations, vol. 111, pp. 1-14, 2009.

[3] A. Caicedo, C. Cuevas, G. M. Mophou, and G. M. N'Guérékata, "Asymptotic behavior of solutions of some semilinear functional differential and integro-differential equations with infinite delay in Banach spaces," Journal of the Franklin Institute: Engineering and Applied Mathematics, vol. 349, no. 1, pp. 1-24, 2012.

[4] T. Diagana, G. Nguerekata, and N. van Minh, "Almost automorphic solutions of evolution equations," Proceedings of the American Mathematical Society, vol. 132, no. 11, pp. 3289-3298, 2004.

[5] T. Diagana, E. M. Hernández, and J. P. C. dos Santos, "Existence of asymptotically almost automorphic solutions to some abstract partial neutral integro-differential equations," Nonlinear Analysis: Theory, Methods \& Applications, vol. 71, no. 1-2, pp. 248-257, 2009.

[6] H.-S. Ding, T.-J. Xiao, and J. Liang, "Asymptotically almost automorphic solutions for some integrodifferential equations with nonlocal initial conditions," Journal of Mathematical Analysis and Applications, vol. 338, no. 1, pp. 141-151, 2008.

[7] J. P. C. dos Santos and C. Cuevas, "Asymptotically almost automorphic solutions of abstract fractional integro-differential neutral equations," Applied Mathematics Letters, vol. 23, no. 9, pp. 960-965, 2010.

[8] Y. J. Liu and A. M. Liu, "Almost periodic solutions for a class of stochastic differential equations," Journal of Computational and Nonlinear Dynamics, vol. 8, no. 4, Article ID 041002, 6 pages, 2013.

[9] G. M. NGuerekata, Almost Automorphic and Almost Periodic Functions in Abstract Spaces, Plenum Publishing Corporation, 2001.

[10] G. M. N'Guérékata, Topics in Almost Automorphy, Springer, New York, NY, USA, 2005.

[11] W. A. Veech, "Almost automorphic functions," Proceedings of the National Academy of Sciences of the United States of America, vol. 49, no. 4, pp. 462-464, 1963.

[12] Z. Huang and Q.-G. Yang, "Existence and exponential stability of almost periodic solution for stochastic cellular neural networks with delay," Chaos, Solitons \& Fractals, vol. 42, no. 2, pp. 773-780, 2009.

[13] J. Cao, Q. Yang, and Z. Huang, "Existence and exponential stability of almost automorphic mild solutions for stochastic functional differential equations," Stochastics, vol. 83, no. 3, pp. 259-275, 2011.

[14] P. H. Bezandry, "Existence of almost periodic solutions to some functional integro-differential stochastic evolution equations," Statistics \& Probability Letters, vol. 78, no. 17, pp. 2844-2849, 2008.

[15] Y.-K. Chang, Z.-H. Zhao, and G. M. N'Guérékata, “A new composition theorem for square-mean almost automorphic functions and applications to stochastic differential equations," Nonlinear Analysis: Theory, Methods \& Applications, vol. 74, no. 6, pp. 2210-2219, 2011. 
[16] W. Desch, R. Grimmer, and W. Schappacher, "Well-posedness and wave propagation for a class of integrodifferential equations in Banach space," Journal of Differential Equations, vol. 74, no. 2, pp. 391-411, 1988.

[17] M. Fu and Z. Liu, "Square-mean almost automorphic solutions for some stochastic differential equations," Proceedings of the American Mathematical Society, vol. 138, no. 10, pp. 3689-3701, 2010.

[18] W. Grecksch and C. Tudor, Stochastic Evolution Equations:A Hilbert Space Approach, Akademie, Berlin, Germany, 1995.

[19] D. N. Keck and M. A. McKibben, "Functional integrodifferential stochastic evolution equations in Hilbert space," Journal of Applied Mathematics and Stochastic Analysis, vol. 16, no. 2, pp. 141-161, 2003.

[20] D. N. Keck and M. A. McKibben, "Abstract stochastic integrodifferential delay equations," Journal of Applied Mathematics and Stochastic Analysis, vol. 2005, no. 3, pp. 275-305, 2005.

[21] H.-S. Ding, J. Liang, and T.-J. Xiao, "Pseudo almost periodic solutions to integro-differential equations of heat conduction in materials with memory," Nonlinear Analysis: Real World Applications, vol. 13, no. 6, pp. 2659-2670, 2012.

[22] R. C. Grimmer, "Resolvent operators for integral equations in a Banach space," Transactions of the American Mathematical Society, vol. 273, no. 1, pp. 333-349, 1982.

[23] G. Chen, "Control and stabilization for the wave equation in a bounded domain," SIAM Journal, vol. 17, no. 1, pp. 66-81, 1979. 


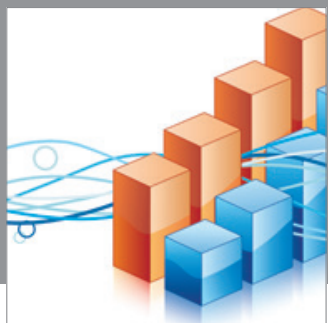

Advances in

Operations Research

mansans

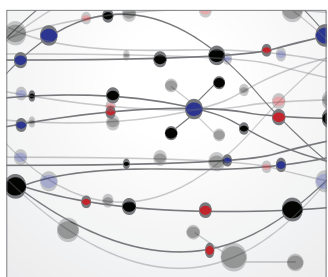

The Scientific World Journal
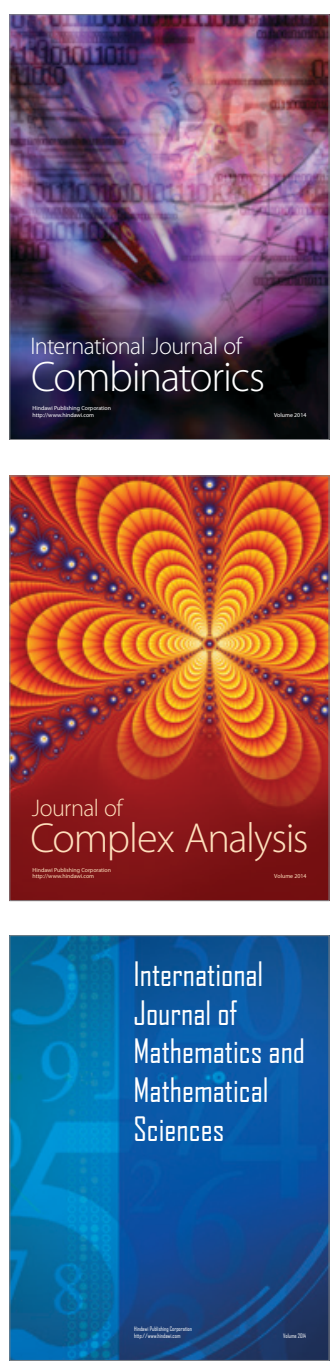
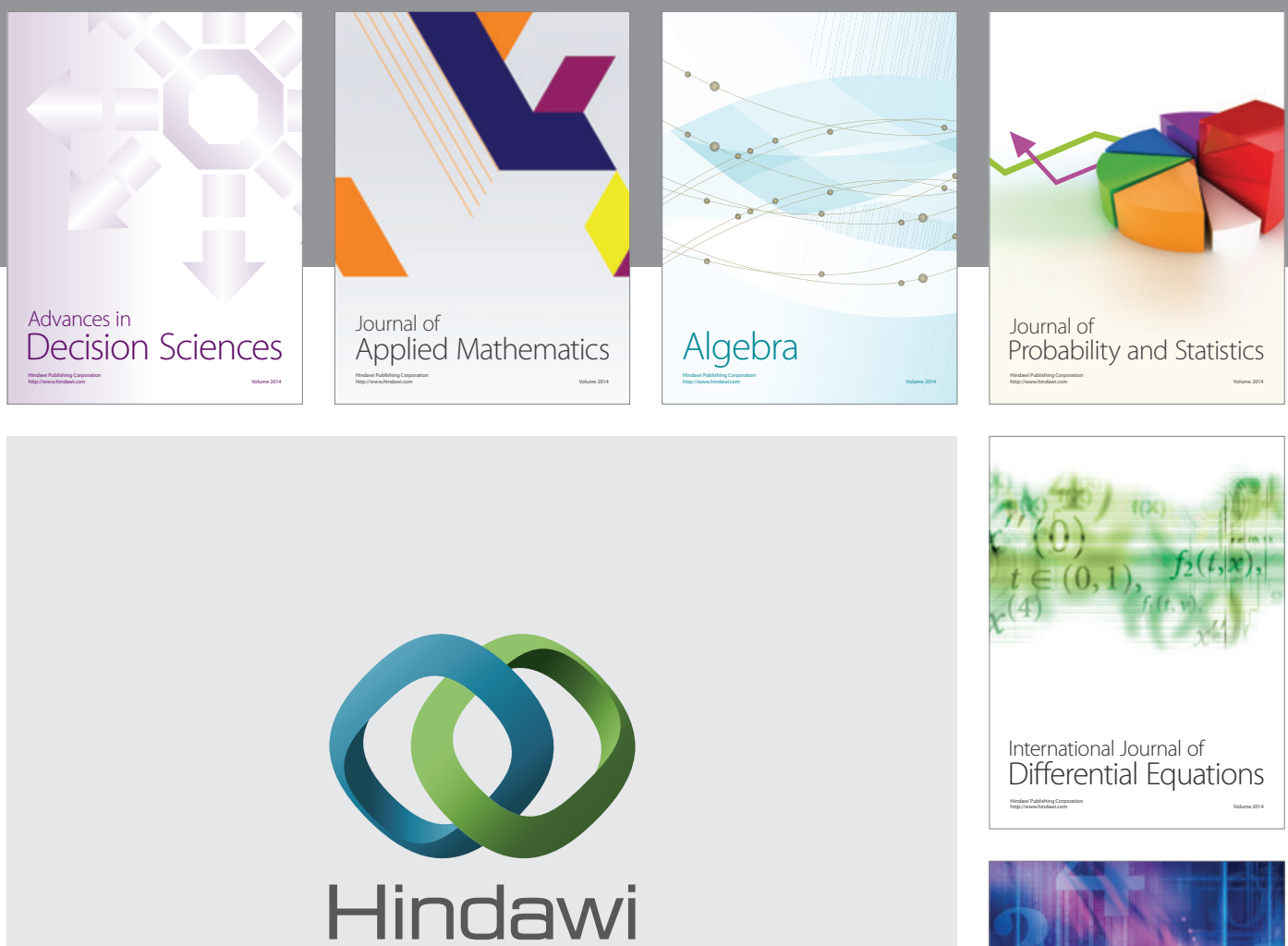

Submit your manuscripts at http://www.hindawi.com


Journal of

Function Spaces

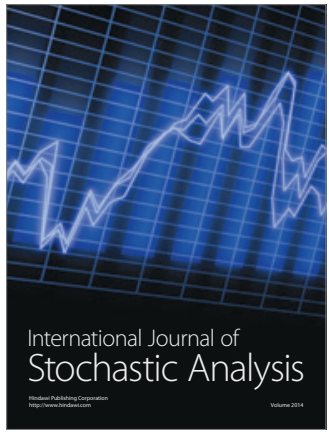

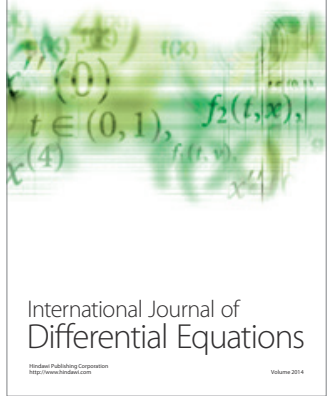
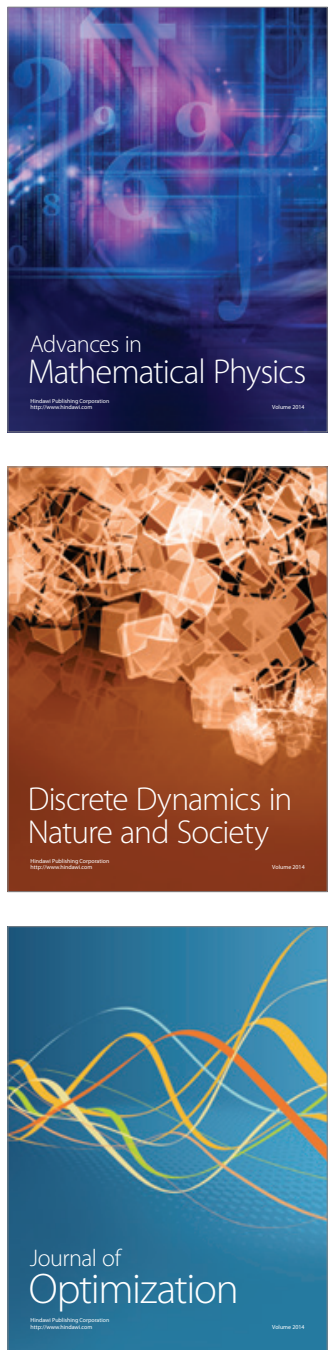